\title{
Impella Device is Superior to an Intra-Aortic Balloon Pump in Cardiogenic Shock
}

\author{
Muhammad Zubair Khan, $M D^{1}$ (D), Sona Franklin, $M D^{1}$ (D) and Richard Hyman, $M D^{2^{*}}$ (i) \\ ${ }^{1}$ Department of Internal Medicine, St. Mary Medical Center, USA \\ ${ }^{2}$ Department of Cardiology, St. Mary Medical Center, USA
}

*Corresponding author: Richard Hyman, MD, Department of Medicine, St. Mary Medical Center, 1201 NewtownLanghorne Rd, Langhorne, PA 19047, USA, Tel: +1-215-750-7000

\begin{abstract}
A Percutaneous assist device is commonly used in cardiogenic shock to improve hemodynamics. The Impella provided superior hemodynamic support in the ISARSHOCK study compared with an intra-aortic balloon pump (IABP), with no change in clinical outcome. In this trial, the IABP was used in one group, and the Impella was placed in a comparator group. We present a case in which the hemodynamics of cardiogenic shock was assessed in the same patient after IABP and after Impella placement. In our case, the hemodynamics improved after Impella placement and removal of IABP
\end{abstract}

\section{Introduction}

Cardiogenic shock (CS) is a common cause of death in acute myocardial infarction. It has been reported that the 30 -day mortality rate is up to $60-80 \%$ in CS $[1,2]$. Intra-aortic balloon pump (IABP) or Impella device are the most common devices used in cardiogenic shock to maintain hemodynamics. IABP was downgraded after the IABP-SHOCK II trial failed to show any mortality benefits over medical therapy $[3,4]$. Impella is an alternative for percutaneous mechanical circulatory support (pMCS), which is widely used as a bridge for recovery. Reports in the literature have shown that the Impella is better than IABP in hemodynamic improvement; however, this improvement was found in different patients and not within the same patients. We report a case in which an Impella was placed after an IABP was removed in the same patient with the goal of improved hemodynamics.

\section{Case Presentation}

A 68-year-old female with a past medical history of systemic lupus erythematosus, transient ischemic attack, and migraine headaches was admitted for heart failure exacerbation. On admission, a physical exam showed rales bilaterally and +2 lower extremity edema bilaterally. The remainder of the physical examination was unremarkable. Blood pressure was $100 / 55 \mathrm{mmHg}$, pulse $110 \mathrm{bpm}$, and all other vital signs were stable. Electrocardiogram (EKG) showed a slight inferior ST elevation in lead III only with Q-waves, a $1 \mathrm{~mm} \mathrm{ST-seg-}$ ment depression in leads I and a VL, and an anterior infarct pattern (Figure 1). An old EKG from 2014 also revealed the anterior infarct pattern; however, the ST segment changes were new. Chest $X$-ray showed diffuse infiltrates bilaterally, and heart size was mildly enlarged (Figure 2). An echocardiogram showed a severely dilated left ventricle and depressed left ventricular systolic function. Left ventricular ejection fraction (LVEF) was $25 \%$, and the total wall motion score was 1.81 .

The Patient's hemodynamics worsened, so an IABP was placed before a heart catheterization. Right and left heart catheterization was performed after IABP placement which showed: A right atrial pressure of $18 \mathrm{mmHg}$, right ventricular pressure was $50 / 18 \mathrm{mmHg}$, pulmonary artery pressure was $52 / 20 \mathrm{mmHg}$, a mean of $42 \mathrm{mmHg}$, and a wedge pressure was $35 \mathrm{mmHg}$ without significant $\checkmark$ waves. Total vascular resistance was $1218 \mathrm{dsc}-5$. The cardiac index was $2.62 \mathrm{l} / \mathrm{min} / \mathrm{m}^{2}$, and cardiac output was $5.58 \mathrm{~L} / \mathrm{min}$. There was a $90 \%$ ostial stenosis, a $50 \%$ mid-

Citation: Khan MZ, Franklin S, Hyman R, Khan MZ, Franklin S, Hyman R (2021) Impella Device is Superior to an Intra-Aortic Balloon Pump in Cardiogenic Shock. Int Arch Cardiovasc Dis 5:040. doi. org/10.23937/2643-3966/1710040

Accepted: July 01, 2021: Published: July 03, 2021

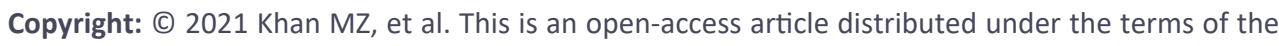
Creative Commons Attribution License, which permits unrestricted use, distribution, and reproduction in any medium, provided the original author and source are credited. 


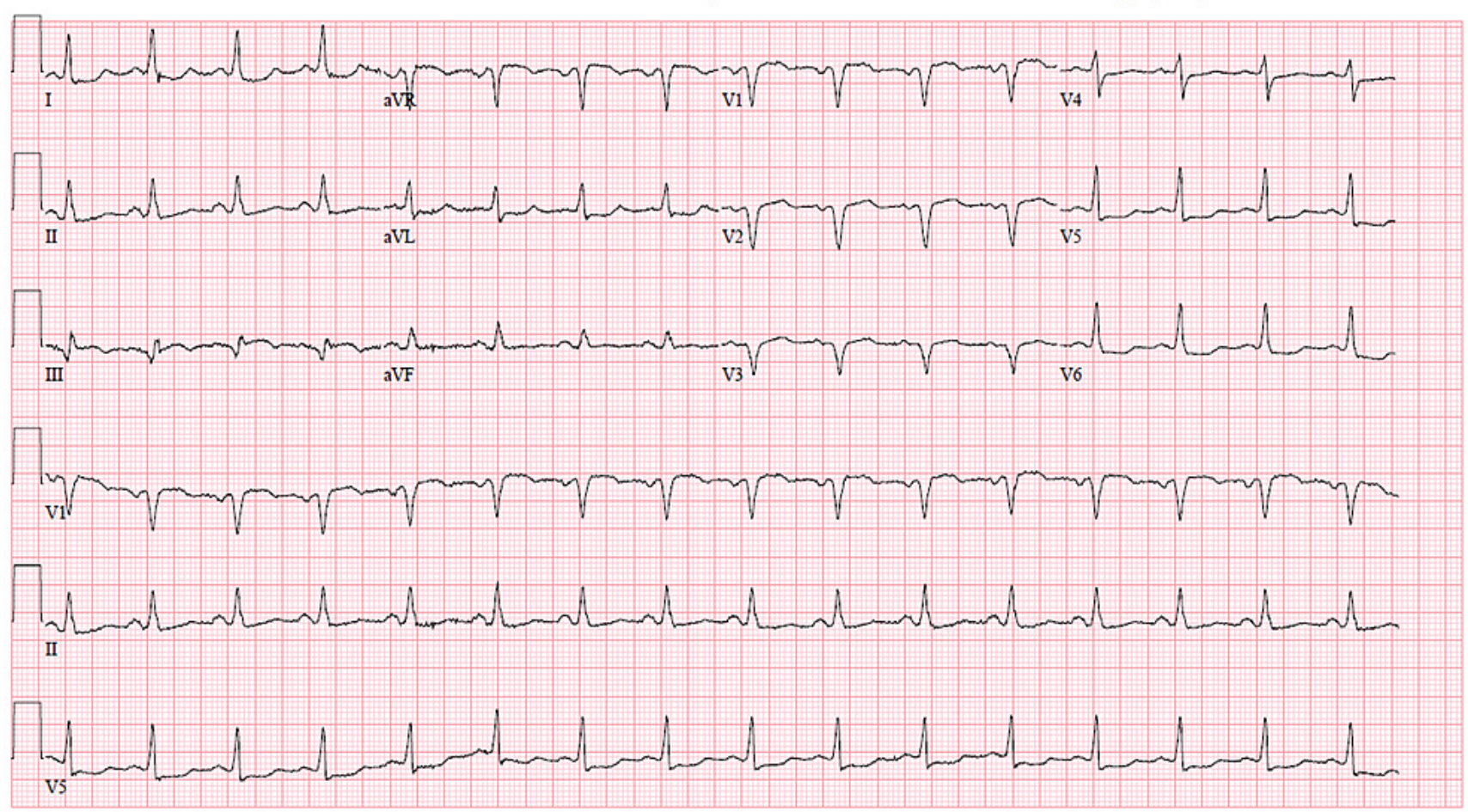

Figure 1: Electrocardiogram showed a slight inferior ST elevation in lead III only with Q-waves, a $1 \mathrm{~mm}$ ST-segment depression in leads I and $\mathrm{aVL}$, and an anterior infarct pattern.

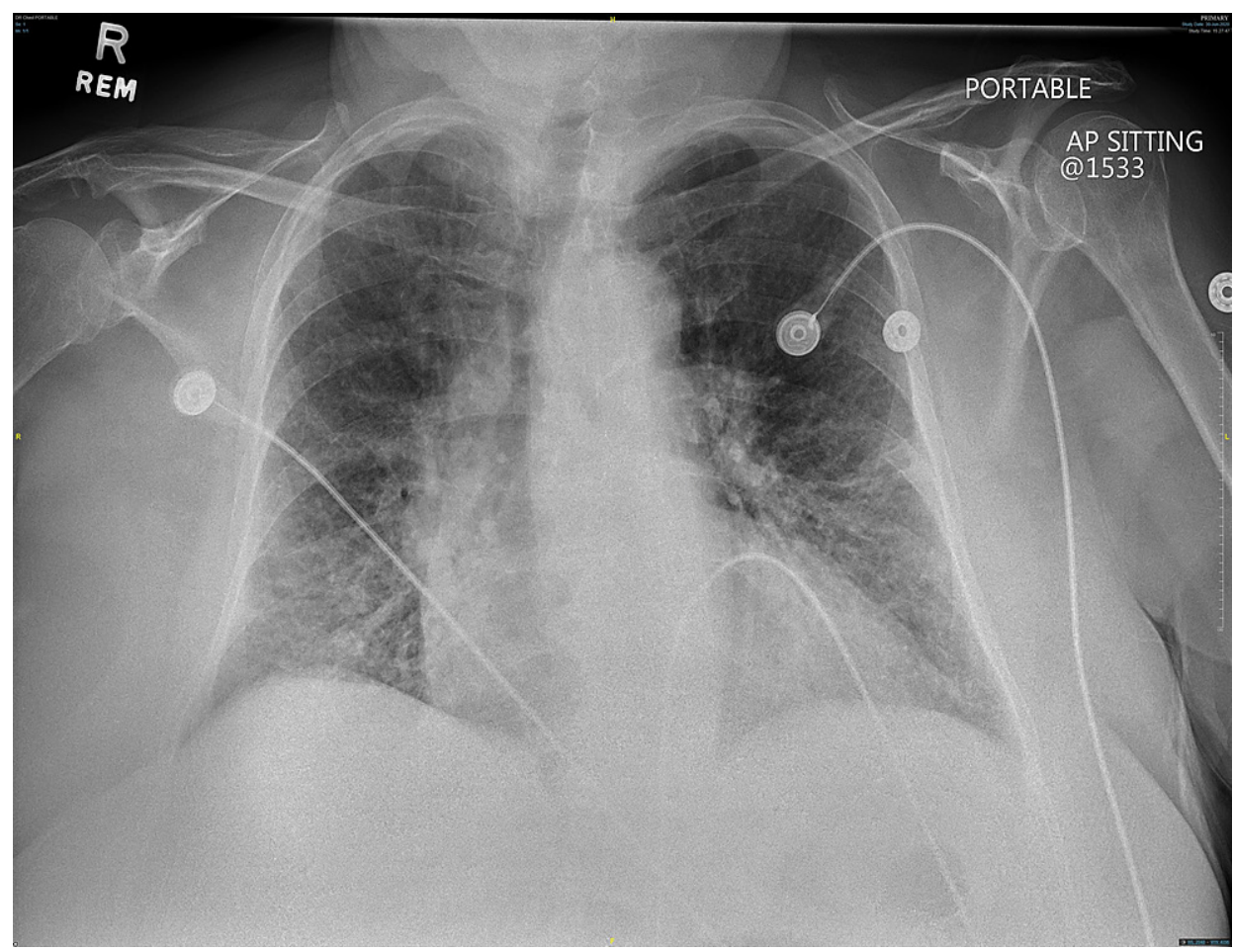

Figure 2: Chest X-ray showed diffuse infiltrates bilaterally.

left anterior descending artery (LAD) lesion with a graftable LAD and diagonal target. After right and left heart catheterization, the Patient started to deteriorate; he developed pulmonary edema and required emergency intubation. A left ventriculogram was performed, which revealed a moderately dilated left ventricular chamber with hypokinesis of the inferior wall, a moderately dilated left ventricular chamber with severe hypokinesis of the inferolateral walls, and mild hypokinesis of the anterior wall. The overall systolic function was impaired with an ejection fraction of $35 \%$. There was a mild $2+$ mitral regurgitation found. The ventriculogram did not suggest acute mitral regurgitation due to papillary muscle rupture. At this point, the Impella device was placed, and IABP was removed. The Patient experienced ventricle fibrillation arrest the following day and required 
shock and cardiopulmonary resuscitation (CPR). The cardiologist adjusted the Impella device under transesophageal echocardiography guidance. Transthoracic echocardiography was performed again the next day, which showed hypokinesis of the entire anterolateral and inferolateral walls with severely depressed ventricular systolic dysfunction left ventricular ejection fraction of $25 \%$. The Patient underwent coronary artery bypass grafting times 3 (left internal mammary artery to LAD, saphenous vein graft to obtuse marginal branch, saphenous vein graft to the posterior descending coronary artery, and endoscopic vein harvest of the left greater saphenous vein). She developed atrial fibrillation (AF) with a rapid ventricular response (AF with RVR) and required four cardioversions at the bedside in a 24-hour period. She was found to be febrile with leukocytosis, which was thought to be due to MSSA associated left basilar pneumonia, and she was treated with cefazolin. She also developed $C$. difficile colitis and was treated with oral vancomycin. The patient developed torsades at the pointes and was cardioverted. Tracheostomy and percutaneous endoscopic gastrostomy tubes were placed. The patient's condition improved steadily, but she was unable to be weaned off mechanical ventilation. Her condition stabilized, and she was eventually cleared for discharge on ventilator support with tube feeds via percutaneous endoscopic gastrostomy tube. The Patient was admitted again nine months later for gastrointestinal bleed and was later discharged to the rehab facility.

\section{Discussion}

Our case described the benefits of an Impella device for improving hemodynamics in CS when IABP was found to be inferior. Previous studies have shown that Impella improves hemodynamics better than IABP; however, this improvement was noted in different patients. In our case, an IABP was placed first, which did not improve hemodynamics. IABP was subsequently removed, and Impella was then placed, which showed improved hemodynamics.

IABP has minimal effects on preload and cannot independently support the systemic circulation in cases of cardiogenic shock. Impella actively unloads the left ventricle into the ascending aorta and reduces the oxygen demand on the left ventricle. If the IABP does not improve hemodynamics in cardiogenic shock, Impella can maintain cardiac output $[5,6]$. Studies have also shown that both devices simultaneously showed superior hemodynamics compared to a single device alone [5].

Despite early revascularization, in acute myocardial infarction complicated by cardiogenic shock (AMICS), the mortality is $40-70 \%$. Impella provides superior hemodynamics to explain the significant reduction of lactate and to lower inotropes used in these patients. However, the length of stay, mechanical ventilation rate, and renal replacement therapy (RRT) were not different in patients treated with IABP [7]. Also, the reduction in 30-day mortality with Impella as compared with IABP or standard medical therapy demonstrated a lack of benefits. Some studies suggested a protective effect exerted by Impella in "lower risk" patients. In the "Detroit Cardiogenic Shock Initiative," the early discontinuation of inotropes and vasopressors and the rapid delivery of a pMCS device within 90 minutes of shock development can improve mortality compared to standard medical therapy. Of note, in that study, lower-risk patients were evaluated [7]. In the IABP-SHOCK II study, neither mortality benefit nor harm in patients with CS-AMI randomized to the addition of an IABP to inotropes. In the ISAR-SHOCK study, the Impella LP2.5 provided early, transitory, and slightly superior hemodynamic support compared with an IABP, with no change in outcome. In the ISAR-SHOCK and IMPRESS trials, in severe shock, the pMCS devices were inserted after percutaneous coronary intervention (PCI) for AMI-CS; thus the 30-day mortality reduction was not statistically significant $[8,9]$. However, a recent study showed that left ventricular unloading before $\mathrm{PCl}$ to prevent reperfusion injury could be helpful $[10,11]$. Therefore, a randomized control trial of Impella 2.5 insertion before reperfusion compared to no MCS is required. The newer generation Impella 5 devices, which are now available, can provide a cardiac output of $5 \mathrm{~L} /$ min. Studies have suggested that Impella 5 provides superior outcomes compared to Impella 2.5 in the setting of AMI-CS. Currently, the Impella 5 is limited in daily use as its large profile requires surgical cut-down. Burkhoff, et al. show the native cardiac output increases as the flow of the pump increases by a mechanical circulatory support device. When Impella is placed, the native output is overcome and most of the flow is from the Impella pump. This kind of mechanism was found in the IMPELLA-STIC randomized study $[12,13]$. In our study, the cardiac output was increased by the impella device. In addition, there is an emerging concept of ECPELLA (venoarterial extracorporeal membrane oxygen [VA-ECMO] and Impella), which would provide a more robust hemodynamic support and is superior to VA-ECMO alone [12]. In our case, the importance of the Impella device implementation was noted in CS as the IABP failed to improve our patient's hemodynamics.

\section{Conclusion}

Impella can be substituted for an IABP when further improvement in hemodynamics support is needed in CS.

\section{Funding}

None declared.

\section{Conflict of Interest}

None declared.

\section{References}

1. Khalid L, Dhakam SH (2008) A review of cardiogenic shock in acute myocardial infarction. Curr Cardiol Rev 4: 34-40. 
2. Antman EM, Anbe DT, Armstrong PW, Bates ER, Green LA, et al. (2004) ACC/ AHA guidelines for the management of patients with ST-elevation myocardial infarction--executive summary. A report of the American College of Cardiology/American Heart Association Task Force on Practice Guidelines. J Am Coll Cardiol 44: 671-719.

3. Kolh P, Windecker S, Alfonso F, Collet JP, Cremer J, et al. (2014) 2014 ESC/EACTS guidelines on myocardial revascularization: The task Force on myocardial revascularization of the. European Society of cardiology (ESC) and the European association for Cardio-Thoracic surgery (EACTS). Developed with the special contribution of the European association of percutaneous cardiovascular interventions (EAPCI). Eur J Cardiothorac Surg 46: 517-592.

4. Thiele H, Zeymer U, Neumann F-J, Ferenc M, Olbrich HG, et al. (2013) Intra-aortic balloon counterpulsation in acute myocardial infarction complicated by cardiogenic shock (IABP-SHOCK II): Final 12-month results of a randomised, open-label trial. Lancet 382: 1638-1645.

5. Gupta A, Allaqaband S, Bajwa T (2009) Combined use of Impella device and intra-aortic balloon pump to improve survival in a patient in profound cardiogenic shock post cardiac arrest. Catheter Cardiovasc Interv 74: 975-976.

6. Sauren LD, Accord RE, Hamzeh K, de Jong M, van der Nagel T, et al. (2007) Combined Impella and intra-aortic balloon pump support to improve both ventricular unloading and coronary blood flow for myocardial recovery: An experimental study. Artif Organs 31: 839-842.

7. Basir MB, Schreiber T, Dixon S, Alaswad K, Kirit Patel K, et al. (2018) Feasibility of early mechanical circulatory support in acute myocardial infarction complicated by cardiogenic shock: The Detroit cardiogenic shock initiative. Catheter Cardiovasc Interv 91: 454-461.
8. Ouweneel DM, Eriksen E, Sjauw KD, Dongen IMV, Hirsch A, et al. (2017) Percutaneous mechanical circulatory support versus intra-aortic balloon pump in cardiogenic shock after acute myocardial infarction. J Am Coll Cardiol 69: 278287.

9. Seyfarth M, Sibbing D, Bauer I, Fröhlich G, Flügel LB, et al. (2008) A randomized clinical trial to evaluate the safety and efficacy of a percutaneous left ventricular assist device versus intra-aortic balloon pumping for treatment of cardiogenic shock caused by myocardial infarction. J Am Coll Cardiol 52: 1584-1588.

10. Kapur NK, Alkhouli MA, DeMartini TJ, Faraz H, George ZH, et al. (2019) Unloading the left ventricle before reperfusion in patients with anterior ST-segment-elevation myocardial infarction. Circulation 139: 337-346.

11. Loehn T, O'Neill WW, Lange B, Pfluecke C, Schweigler T, et al. (2020) Long term survival after early unloading with Impella $\mathrm{CP}{ }^{\circledR}$ in acute myocardial infarction complicated by cardiogenic shock. Eur Heart J Acute Cardiovasc Care 9: 149-157.

12. Patel SM, Lipinski J, Al-Kindi SG, Patel T, Saric $P$, et al. (2019) Simultaneous Venoarterial extracorporeal membrane oxygenation and percutaneous left ventricular decompression therapy with Impella is associated with improved outcomes in refractory cardiogenic shock. ASAIO J 65: 21-28.

13. Bochaton T, Huot L, Elbaz M, Delmas C, Aissaoui N, et al. (2020) Mechanical circulatory support with the Impella $\circledR$ LP5.0 pump and an intra-aortic balloon pump for cardiogenic shock in acute myocardial infarction: The IMPELLA-STIC randomized study. Arch Cardiovasc Dis 113: 237-243. 